\title{
Ocorrência de carrapatos em animais silvestres recebidos e atendidos pelo Parque Zoológico Municipal Quinzinho de Barros, Sorocaba, São Paulo, Brasil
}

\section{Occurrence of ticks on wild animals received and attended at the Parque Zoológico Municipal Quinzinho de Barros, Sorocaba, São Paulo, Brazil}

\author{
Thiago Fernandes MARTINS ${ }^{1}$; Rodrigo Hidalgo Friciello TEIXEIRA²; Marcelo Bahia LABRUNA ${ }^{1}$ \\ ${ }^{1}$ Universidade de São Paulo, Faculdade de Medicina Veterinária e Zootecnia, \\ Departamento de Medicina Veterinária Preventiva e Saúde Animal, São Paulo - SP, Brasil \\ ${ }^{2}$ Parque Zoológico Municipal Quinzinho de Barros, Sorocaba - SP, Brasil
}

\begin{abstract}
Resumo
Os carrapatos são ectoparasitas da classe Arachnida que parasitam vertebrados terrestres, anfíbios, répteis, aves e mamíferos. O presente trabalho relata a ocorrência de carrapatos ixodídeos em animais silvestres recebidos e atendidos pelo Hospital Veterinário do Parque Zoológico Municipal Quinzinho de Barros, localizado no município de Sorocaba, estado de São Paulo, Brasil. De setembro de 1999 a maio de 2015, foram coletados carrapatos em animais silvestres da região de Sorocaba e de outros 20 municípios do interior do estado de São Paulo. Ao todo, foram identificados 43 larvas, 637 ninfas e 1.178 adultos (631 machos e 547 fêmeas), totalizando 1.858 exemplares de 14 espécies distintas de ixodídeos. Durante exames clínicos de rotina, foram inspecionadas duas espécies de répteis, uma espécie de ave e 11 espécies distintas de mamíferos de um total de 103 animais silvestres amostrados. Nos répteis foram identificados Amblyomma rotundatum, nas aves Amblyomma sculptum e nos mamíferos Amblyomma aureolatum, Amblyomma brasiliense, Amblyomma calcaratum, Amblyomma dubitatum, Amblyomma longirostre, Amblyomma nodosum, Amblyomma ovale, A. sculptum, Amblyomma varium, Ixodes aragaoi, Haemaphysalis juxtakochi, Rhipicephalus microplus e Dermacentor nitens. Este estudo relata os primeiros registros de fêmeas de A. rotundatum parasitando Hydromedusa tectifera e Oxyrhopus guibei, assim como ninfas de A. dubitatum e H. juxtakochi em Chrysocyon brachyurus, ninfas de A. brasiliense em Myrmecophaga tridactyla e Tamandua tetradactyla, além de ninfas de A. sculptum em Alouatta guariba e Sphiggurus villosus no país, demonstrando que os zoológicos são uma fonte de informação valiosa para o conhecimento parasitológico da fauna silvestre brasileira.
\end{abstract}

Palavras-chave: Carrapatos. Animais Silvestres. Zoológico. São Paulo. Brasil.

\begin{abstract}
Ticks are ectoparasites of the class Arachnida that parasitize terrestrial vertebrates, amphibians, reptiles, birds and mammals. This paper reports the occurrence of hard ticks on wild animals received and attended by the Veterinary Hospital of "Parque Zoológico Municipal Quinzinho de Barros", located in Sorocaba municipality, São Paulo state, Brazil. From September 1999 to May 2015, ticks were collected on wild animals of the Sorocaba region and other 20 municipalities in the state of São Paulo. Altogether, 43 larvae, 637 nymphs and 1,178 adults (631 males and 547 females) were identified, totaling 1,858 samples of 14 different tick species. During routine clinical examinations, two species of reptiles, a species of bird and 11 different species of mammals were inspected, resulting in 103 samples from wild animals. The following tick species were found: Amblyomma rotundatum on reptiles; Amblyomma sculptum on birds; and Amblyomma aureolatum, Amblyomma brasiliense, Amblyomma calcaratum, Amblyomma dubitatum, Amblyomma longirostre, Amblyomma nodosum, Amblyomma ovale, A. sculptum, Amblyomma varium, Ixodes aragaoi, Haemaphysalis juxtakochi, Rhipicephalus microplus and Dermacentor nitens on mammals. This study reports the first records of $A$. rotundatum females parasitizing Hydromedusa tectifera and Oxyrhopus guibei, A. dubitatum nymphs and $H$. juxtakochi nymphs on Chrysocyon brachyurus, A. brasiliense nymphs on Myrmecophaga tridactyla and Tamandua tetradactyla, and A. sculptum nymphs on Alouatta guariba and Sphiggurus villosus. Our results highlight zoos as a source of valuable information for the parasitological knowledge of Brazilian wildlife.
\end{abstract}

Keywords: Ticks. Wild Animals. Zoo. São Paulo. Brazil. 


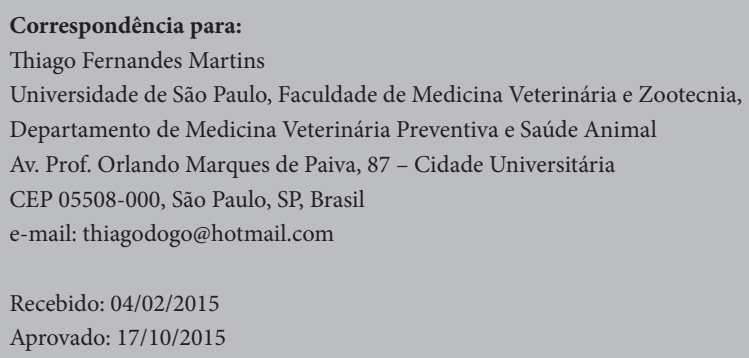

\section{Introdução}

Os carrapatos são ectoparasitas da classe Arachnida, de distribuição mundial, que parasitam animais domésticos, silvestres e acidentalmente humanos (ARAGÃO, 1936). Devido à hematofagia, os carrapatos são o primeiro grupo em importância de vetores de agentes infecciosos para animais e o segundo para humanos, ficando apenas atrás dos mosquitos. Entre os agentes transmitidos, incluem-se vírus, bactérias, protozoários e helmintos (GUIMARÃES; TUCCI; BARROS-BATTESTI, 2001). As espécies de carrapatos que parasitam animais domésticos são bastante estudadas, enquanto aquelas que parasitam animais silvestres são pouco conhecidas (BARROS-BATTESTI; ARZUA; BECHARA, 2006).

O Parque Zoológico Municipal Quinzinho de Barros ocupa uma área de $128.000 \mathrm{~m}^{2}$ e está localizado no município de Sorocaba $\left(23^{\circ} 30^{\prime} \mathrm{S} 47^{\circ} 30^{\prime} \mathrm{W}\right)$ interior do estado de São Paulo, Brasil. O local é uma referência no atendimento emergencial e tratamento de animais silvestres da região de Sorocaba e municípios vizinhos. Além de cuidar da saúde e bem-estar dos animais selvagens que residem no Parque Zoológico, os funcionários recebem e atendem inúmeras espécies da fauna do estado de São Paulo, incluindo as raras, endêmicas e/ou ameaçadas de extinção, contribuindo, desse modo, na conservação da biodiversidade faunística da região (TEIXEIRA, 2001).

Devido à importância do conhecimento dos carrapatos que parasitam a fauna silvestre brasileira, o objetivo deste trabalho foi relatar a ocorrência de ixodídeos em animais silvestres recebidos e atendidos no
Zoológico de Sorocaba, durante exames clínicos de rotina.

\section{Materiais e Métodos}

Os carrapatos dos animais silvestres foram coletados entre os meses de setembro de 1999 a maio de 2015, sendo os hospedeiros provenientes da região de Sorocaba e de outros 20 municípios do interior do estado de São Paulo (Tabela 1). Todos os animais foram resgatados e encaminhados para o Zoológico pela população local, Instituto Brasileiro do Meio Ambiente e dos Recursos Naturais Renováveis (IBAMA), Instituto Chico Mendes de Conservação da Biodiversidade (ICMBio), $3^{\text {a }}$ Companhia da Polícia Militar Ambiental, Guarda Civil Metropolitana e $15^{\circ}$ Grupamento de Bombeiros; em geral, animais silvestres encontrados feridos devido a maus tratos, acidentes causados pela ação antrópica, indivíduos jovens e até mesmo filhotes.

Os animais inspecionados pertenciam a diferentes faixas etárias e sexos, sendo que os mesmos foram identificados pelos técnicos do Zoológico de Sorocaba.

No Setor Veterinário do Parque Zoológico, os carrapatos fixados foram detectados por inspeção na pele dos animais silvestres, figuras 1 e 2, e, desse modo, foram cuidadosamente removidos manualmente. Os ixodídeos coletados foram armazenados vivos em frascos tipo "coletor universal" e enviados para o Laboratório de Doenças Parasitárias da Faculdade de Medicina Veterinária e Zootecnia da Universidade de São Paulo. Alguns carrapatos coletados nos hospedeiros como ninfas ingurgitadas, foram colocados em estufa tipo B.O.D. (demanda bioquímica de oxigênio) na temperatura de $25^{\circ} \mathrm{C}$ e umidade relativa de $85 \%$, para a ecdise da fase adulta em condições de laboratório, segundo Labruna et al. (2002). Os exemplares foram identificados utilizando estereomicroscópio com iluminação incidente, chaves taxonômicas e literatura correspondente (ARAGÃO; FONSECA, 1961; GUIMARÃES; TUCCI; BARROS-BATTESTI, 2001; ONOFRIO et al., 2006; MARTINS et al., 2010; NAVA et al., 2014). Todos os espécimes foram depositados 


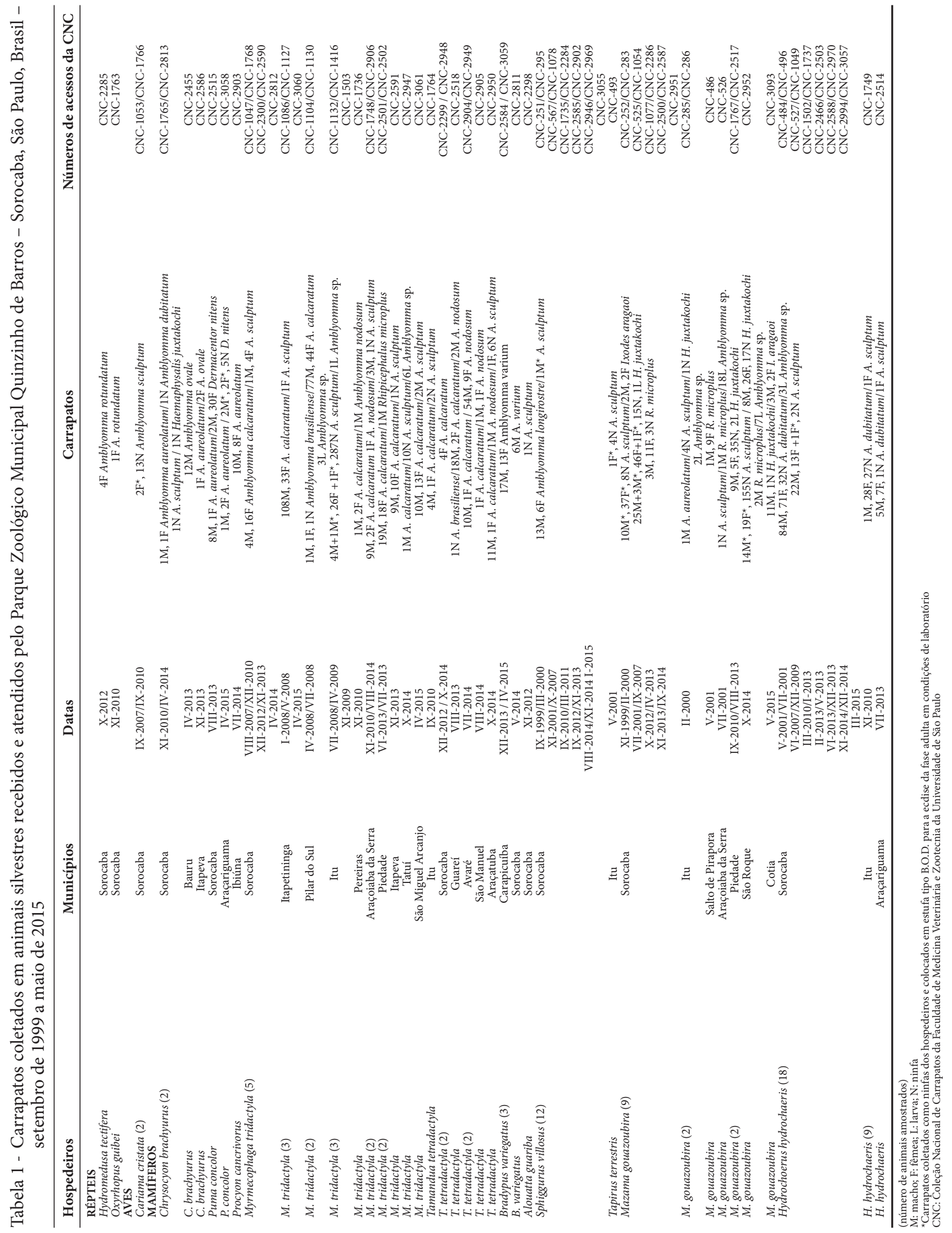


na Coleção Nacional de Carrapatos da Faculdade de Medicina Veterinária e Zootecnia da Universidade de São Paulo.

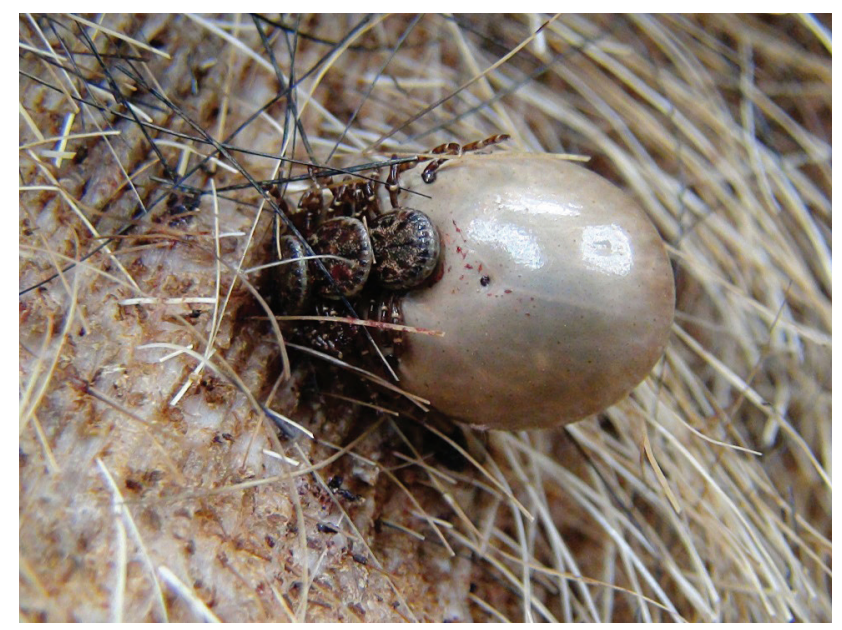

Figura 1 - Amblyomma calcaratum parasitando MyrFonte: (TEIXEIRA, 2014)

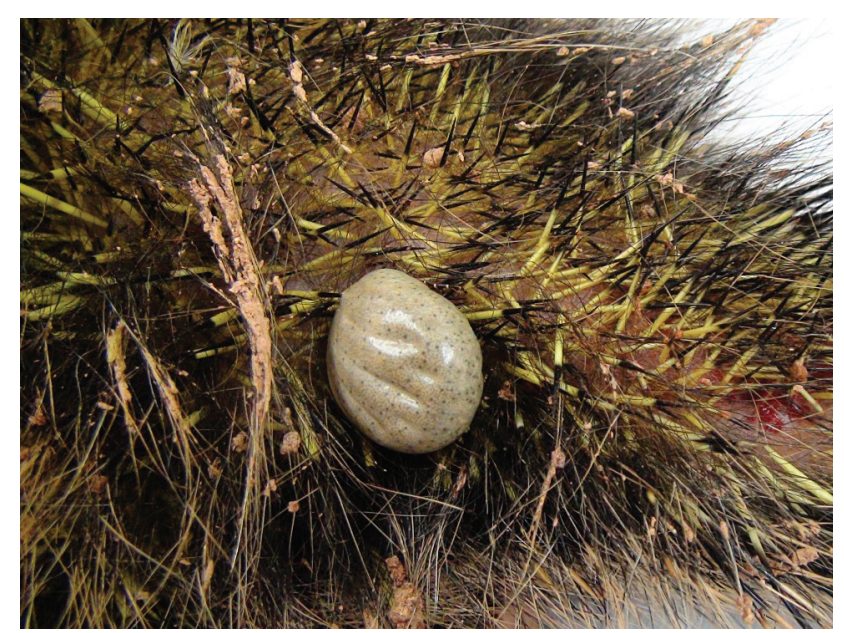

Figura 2 - Amblyomma longirostre parasitando Sphiggurus villosus

Fonte: (TEIXEIRA, 2014)

\section{Resultados e Discussão}

Foram amostrados dois répteis: cágado-pescoço-de-cobra (Hydromedusa tectifera) e falsa-coral (Oxyrhopus guibei); duas aves: seriema (Cariama cristata), e 99 mamíferos: sendo quatro lobos-guará (Chrysocyon brachyurus), duas onças-pardas (Puma concolor), um mão-pelada (Procyon cancrivorus),
21 tamanduás-bandeira (Myrmecophaga tridactyla), oito tamanduás-mirins (Tamandua tetradactyla), quatro preguiças-comuns (Bradypus variegatus), um bugio-ruivo (Alouatta guariba), 12 ouriços-cacheiros (Sphiggurus villosus), uma anta (Tapirus terrestris), 17 veados-catingueiros (Mazama gouazoubira) e 28 capivaras (Hydrochoerus hydrochaeris). Ao todo, foram identificados 43 larvas, 637 ninfas e 1.178 adultos (631 machos e 547 fêmeas), totalizando 1.858 exemplares de 14 espécies distintas de ixodídeos (Tabela 1).

Das associações parasita-hospedeiro encontradas no presente estudo, destacam-se os primeiros relatos nunca antes publicados de fêmeas de Amblyomma rotundatum parasitando $H$. tectifera e O. guibei no território nacional. $\mathrm{O}$ carrapato $A$. rotundatum é a espécie mais encontrada em répteis e anfíbios brasileiros em condições naturais e, eventualmente, em cativeiro, sendo que há um registro de hiperparasitismo nesta última condição (LABRUNA et al., 2007a). Os hospedeiros dessa espécie são animais pecilotérmicos, sendo raramente observada em animais homeotérmicos (GUGLIELMONE; NAVA, 2010). É um ixodídeo que se reproduz partenogeneticamente, embora haja dois relatos de machos. (LABRUNA; TERASSINI; CAMARGO, 2005; MARTINS et al., 2014).

Neste trabalho, foram encontradas 13 ninfas e duas fêmeas (que foram coletadas como ninfas) de Amblyomma sculptum em dois indivíduos de C. cristata, ambos do município de Sorocaba. Esses achados corroboram o estudo prévio de Labruna et al. (2007), que relataram uma ninfa dessa espécie de carrapato (publicado como Amblyomma cajennense) parasitando uma ave $C$. cristata no município de Jundiaí, interior do estado de São Paulo.

No presente estudo, foram encontradas, pela primeira vez no país, uma ninfa de Amblyomma dubitatum e uma ninfa de Haemaphysalis juxtakochi em dois C. brachyurus do município de Sorocaba. Entretanto Labruna et al. (2005) encontraram apenas um adulto de A. dubitatum parasitando Cerdocyon thous em Jordanésia, estado de São Paulo, e não encontraram ne- 
nhum carrapato da espécie $H$. juxtakochi parasitando carnívoros silvestres no Brasil.

O presente trabalho registra, pela primeira vez no território brasileiro, duas ninfas de Amblyomma brasiliense em M. tridactyla e T. tetradactyla, nos municípios de Pilar do Sul e Guareí, respectivamente. As demais espécies de carrapatos encontradas no presente estudo já foram relatadas recentemente nesses dois hospedeiros, incluindo adultos de $A$. brasiliense em M. tridactyla por Teixeira e Miranda (2012) e a espécie A. sculptum (publicado como A. cajennense) por Labruna et al. (2002).

Neste estudo, foram encontradas duas ninfas de $A$. sculptum em um A. guariba e em um S. villosus, pela primeira vez no país. Por outro lado, Labruna et al. (2002) encontraram 18 ninfas dessa mesma espécie de ixodídeo (publicado como A. cajennense) em três Alouatta caraya provenientes da região da Usina $\mathrm{Hi}$ drelétrica de Porto Primavera.

As demais associações parasita-hospedeiro (Tabela 1) foram previamente relatadas em diferentes trabalhos conduzidos em diversos locais do Brasil e América do Sul (BARROS; BAGGIO, 1992; IVANCOVICH; LUCIANI, 1992; SERRA-FREIRE et al., 1996; LABRUNA et al., 2002; MARQUES et al., 2002; GUGLIELMONE et al., 2003; LABRUNA et al., 2005;

\section{Referências}

ARAGĂO, H. B. Ixodidas brasileiros e de alguns paizes limitrophes. Memórias do Instituto Oswaldo Cruz, v. 31, n. 4, p. 759-843, 1936. Disponível em: <http://www.scielo.br/scielo. php? script $=$ sci_arttext\&pid $=S 0074-02761936000400004 \& \operatorname{lng}=\mathrm{pt}$ $\& n r m=i s o \& t l n g=p t>$. Acesso em: 4 nov. 2015. doi: http://dx.doi. org/10.1590/S0074-02761936000400004.

ARAGĂO, H. B.; FONSECA, F. Notas de Ixodologia: VIII. Lista e chave para os representantes da fauna ixodológica brasileira: notas de ixolodogia. Memórias do Instituto Oswaldo Cruz, v. 59, n. 2, p. 115-155, 1961. Disponível em: <http://www.scielo.br/ scielo.php?script=sci_arttext\&pid=S0074-02761961000200001 >. Acesso em: 4 nov. 2015. doi: http://dx.doi.org/10.1590/S007402761961000200001 .

BARROS, D. M.; BAGGIO, D. Ectoparasites Ixodida Leach, 1817 on wild mammals in the State of Paraná, Brazil. Memórias do Instituto Oswaldo Cruz, v. 87, n. 2, p. 291-296, 1992. Disponível em: <http://www.scielo.br/scielo.php?script=sci_arttext\&pid $=$ S0074-02761992000200018 $\mathrm{s}$. Acesso em: 4 nov. 2015. doi: http://dx.doi.org/10.1590/S0074-02761992000200018.

BARROS-BATTESTI, D. M.; ARZUA, M; BECHARA, G. H. Carrapatos de importância médico-veterinária da região
PACHECO et al., 2009; MARTINS et al., 2011), inclusive um estudo que amostrou animais selvagens no próprio Zoológico de Sorocaba (TEIXEIRA, 2001).

Teixeira (2001) e Teixeira e Miranda (2012) demostraram a necessidade da inspeção dos animais silvestres recém-chegados da natureza aos zoológicos, assim como o período de quarentena. O presente trabalho está de acordo com esses dois estudos, reforçando a importância tanto da inspeção quanto da quarentena, ajudando, desse modo, no controle dos carrapatos no plantel de animais desses locais.

Os animais silvestres amostrados durante este trabalho apresentaram uma diversidade de espécies de carrapatos, demostrando que os zoológicos são uma fonte de informação valiosa para o conhecimento parasitológico da fauna silvestre brasileira.

\section{Agradecimentos}

Os autores agradecem Alberto A. Guglielmone (INTA, Rafaela, Argentina) por fornecer dados de seus extensos arquivos de carrapatos da região neotropical. O presente trabalho recebeu auxílio financeiro da Fundação de Amparo à Pesquisa do Estado de São Paulo (FAPESP) e ao Conselho Nacional de Desenvolvimento Científico e Tecnológico (CNPq).

neotropical: um guia ilustrado para identificação de espécies. São Paulo: Vox/ICTTD-3/Butantan, 2006. 223 p.

GUGLIELMONE, A. A.; ESTRADA-PEÑA, A.; MANGOLD, A. J.; BARROS-BATTESTI, D. M.; LABRUNA, M. B.; MARTINS, J. R.; VENZAL, J. M.; ARZUA, M.; KEIRANS, J. E. Amblyomma aureolatum (Pallas, 1772) and Amblyomma ovale Kock, 1844: hosts, distribution and 16S rDNA sequences. Veterinary Parasitology, v. 113 , n. $3-4$, p. $273-288,2003$. Disponível em: <http://www. sciencedirect.com/science/article/pii/S0304401703000839>. Acesso em: 4 nov. 2015. doi: http://dx.doi.org/10.1016/S03044017(03)00083-9.

GUGLIELMONE, A. A.; NAVA, S. Hosts of Amblyomma dissimile Koch, 1844 and Amblyomma rotundatum Koch, 1844 (Acari: Ixodidae). Zootaxa, v. 2541, p. 27-49, 2010.

GUIMARÃES, J. H.; TUCCI, E. C.; BARROS-BATTESTI, D. M. Ectoparasitas de importância veterinária. São Paulo: Editora Plêiade/FAPESP, 2001. p. 52-104.

IVANCOVICH, J. C.; LUCIANI, C. A. Las garrapatas de Argentina. Buenos Aires: Asociación Argentina de Parasitología Veterinaria, $1992.95 \mathrm{p}$.

LABRUNA, M. B.; AHID, S. M. M.; SOARES, H. S.; SUASSUNA, A. C. D. Hyperparasitism in Amblyomma rotundatum (Acari: 
Ixodidae). The Journal of Parasitology, v. 93, n. 6, p. 1531-1532, 2007a. Disponível em: <http://www.bioone.org/doi/abs/10.1645/ GE-1277.1>. Acesso em: 4 nov. 2015. doi: http://dx.doi. org/10.1645/GE-1277.1.

LABRUNA, M. B.; JORGE, R. S. P.; SANA, D. A.; JÁCOMO, A. T. A.; KASHIVAKURA, C. K.; FURTADO, M. M.; FERRO, C.; PEREZ, S. A.; SILVEIRA, L.; SANTOS JR., T. S.; MARQUES, S. R.; MORATO, R. G.; NAVA, A.; ADANIA, C. H.; TEIXEIRA, R. H. F.; GOMES, A. A. B.; CONFORTI, V. A.; AZEVEDO, F. C. C.; PRADA, C. S.; SILVA, J. C. R.; BATISTA, A. F.; MA RV U LO, M. F. V.; MORATO, R. L. G.; ALHO, C. J. R.; PINTER, A.; FERREIRA, P. M.; FERREIRA, F.; BARROS-BATTESTI, D. M. Ticks (Acari: Ixodida) on wild carnivores in Brazil. Experimental and Applied Acarology, v. 36, n. 1-2, p. 149-163, 2005. Disponível em: <http://link.springer.com/article/10.1007\%2 Fs10493-005-2563-1>. Acesso em: 4 nov. 2015. doi: http://dx.doi. org/10.1007/s10493-005-2563-1.

LABRUNA, M. B.; PAULA, C. D.; LIMA, T. F.; SANA, D. A. Ticks (Acari: Ixodidae) on wild animals from the Porto Primavera Hydroelectric power station area, Brazil. Memórias do Instituto Oswaldo Cruz, v. 97, n. 8, p. 1133-1136, 2002. Disponível em: $\quad<$ http://www.scielo.br/scielo.php?script=sci_arttext\&pid $=$ S0074-02762002000800012>. Acesso em: 4 nov. 2015. doi: http://dx.doi.org/10.1590/S0074-02762002000800012.

LABRUNA, M. B.; SANFILIPPO, L. F.; DEMETRIO, C.; MENEZES, A. C.; PINTER, A.; GUGLIELMONE, A. A.; SILVEIRA, L. F. Ticks collected on birds in the State of São Paulo, Brazil. Experimental and Applied Acarology, v. 43, n. 2, p. 147-160, 2007. Disponível em: <http://link.springer.com/ article/10.1007\%2Fs10493-007-9106-x>. Acesso em: 4 nov. 2015. doi: http://dx.doi.org/10.1007/s10493-007-9106-x.

LABRUNA, M. B.; TERASSINI, F. A.; CAMARGO, L. M. First report of the male of Amblyomma rotundatum (Acari: Ixodidae) from a field-collected host. Journal of Medical Entomology, v. 42, n. 6, p. 945-947, 2005. Disponível em: <http://www.bioone. org/doi/abs/10.1603\%2F0022-2585(2005)042\%5B0945\%3AFR OTMO\%5D2.0.CO\%3B2>. Acesso em: 4 nov. 2015. doi: http:// dx.doi.org/10.1603/0022-2585(2005)042[0945:FROTMO]2.0. $\mathrm{CO} ; 2$.

MARQUES, S.; BARROS-BATTESTI, D. M.; FACCINI, J. L. H.; ONOFRIO, V. C. Brazilian distribution of Amblyomma varium Koch, 1844 (Acari: Ixodidae), a common parasite of sloths (Mammalia: Xenarthra). Memórias do Instituto Oswaldo Cruz, v. 97, n. 8, p. 1141-1146, 2002. Disponível em: <http://www. scielo.br/scielo.php?pid=S0074-02762002000800014\&script=sci arttext>. Acesso em: 4 nov. 2015. doi: http://dx.doi.org/10.1590/ S0074-02762002000800014.

MARTINS, T. F.; FURTADO, M. M.; JÁCOMO, A. T. A.; SILVEIRA, L.; SOLLMANN, R.; TÔRRES, N. M.; LABRUNA, M. B. Ticks on free-living wild mammals in Emas National Park, Goiás State, central Brazil. Systematic \& Applied Acarology, v. 16, n. 3, p. 201-206, 2011.
MARTINS, T. F.; ONOFRIO, V. C.; BARROS-BATTESTI, D. M.; LABRUNA, M. B. Nymphs of the genus Amblyomma (Acari: Ixodidae) of Brazil: descriptions, redescriptions, and identification key. Ticks and Tick-borne Diseases, v. 1, n. 2, p. 75-99, 2010. Disponível em: <http://www.sciencedirect.com/science/article/ pii/S1877959X10000348>. Acesso em: 4 nov. 2015. doi: http:// dx.doi.org/10.1016/j.ttbdis.2010.03.002.

MARTINS, T. F.; VENZAL, J. M.; TERASSINI, F. A.; COSTA, F. B.; MARCILI, A.; CAMARGO, L. M.; BARROS-BATTESTI, D. M.; LABRUNA, M. B. New tick records from the state of Rondônia, western Amazon, Brazil. Experimental and Applied Acarology, v. 62, n. 1, p. 121-128, 2014. Disponível em: <http://link.springer. com/article/10.1007\%2Fs10493-013-9724-4>. Acesso em: 4 nov. 2015. doi: http://dx.doi.org/10.1007/s10493-013-9724-4.

NAVA, S.; BEATI, L.; LABRUNA, M. B.; CÁCERES, A. G.; MANGOLD, A. J.; GUGLIELMONE, A. A. Reassessment of the taxonomic status of Amblyomma cajennense (Fabricius, 1787) with the description of three new species, Amblyomma tonelliae n. sp., Amblyomma interandinum n. sp. and Amblyomma patinoi n. sp., and reinstatement of Amblyomma mixtum Koch, 1844 and Amblyomma sculptum Berlese, 1888 (Ixodida: Ixodidae). Ticks and Tick-borne Diseases, v. 5, n. 3, p. 252-276, 2014. Disponível em: <http://www.sciencedirect.com/science/article/ pii/S1877959X14000065>. Acesso em: 4 nov. 2015. doi: http:// dx.doi.org/10.1016/j.ttbdis.2013.11.004.

ONOFRIO, V. C.; VENZAL, J. M.; PINTER, A.; SZABÓ, M. P. J. Família Ixodidae: características gerais, comentários e chave para gêneros. In: BARROS-BATTESTI, D. M.; ARZUA, M.; BECHARA G. H. (Ed.). Carrapatos de importância médico-veterinária da região neotropical: um guia ilustrado para identificação de espécies. São Paulo: Vox/ICTTD-3/Butantan, 2006. p. 29-39.

PACHECO, R. C.; HORTA, M. C.; PINTER, A.; MORAES-FILHO, J.; MARTINS, T. F.; NARDI, M. S.; SOUZA, S. S. A.; SOUZA, C. E.; SZABÓ, M. P. J.; RICHTZENHAIN, L. J.; LABRUNA, M. B. Pesquisa de Rickettsia spp. em carrapatos Amblyomma cajennense e Amblyomma dubitatum no Estado de São Paulo. Revista da Sociedade Brasileira de Medicina Tropical, v. 42, n. 3, p. 351-353, 2009. Disponível em: <http://www.scielo.br/ scielo.php?pid=S0037-86822009000300023\&script $=$ sci_arttext $>$. Acesso em: 4 nov. 2015. doi: http://dx.doi.org/10.1590/S003786822009000300023 .

SERRA-FREIRE, N. M.; AMORIM, M.; GAZÊTA, G. S.; GUERIM, L.; DESIDERIO, M. G. H. Ixodofauna de cervídeos no Brasil. Revista Brasileira de Ciência Veterinária, v. 3, n. 2, p. 5154, 1996.

TEIXEIRA, R.; MIRANDA, F. Ixodofauna em tamanduás. In: MIRANDA, F. Manutenção de tamanduás em cativeiro. São Paulo: Cubo, 2012. p. 256-261.

TEIXEIRA, R. H. F. Análise da composição faunística de carrapatos em animais cativos no Zoológico de Sorocaba - SP. 2001. 62 f. Dissertação (Mestrado em Biologia Animal) - Instituto de Biologia, Universidade Federal Rural do Rio de Janeiro, Seropédica, 2001. 